\title{
Normal mode simulation of prompt elastogravity signals induced by an earthquake rupture
}

\author{
K. Juhel ${ }^{\oplus},{ }^{1,2,3}$ J.-P. Montagner, ${ }^{1}$ M. Vallée, ${ }^{1}$ J.P. Ampuero ${ }^{\oplus},{ }^{4,5}$ M. Barsuglia, ${ }^{2}$ P. Bernard, ${ }^{1}$ \\ E. Clévédé, ${ }^{1}$ J. Harms ${ }^{6,7}$ and B.F. Whiting ${ }^{\oplus 8}$ \\ ${ }^{1}$ Institut de Physique du Globe de Paris, Paris 75238, France.E-mail: juhel@ipgp.fr \\ ${ }^{2}$ AstroParticule et Cosmologie, Université Paris Diderot, Paris 75013, France \\ ${ }^{3}$ Université Sorbonne Paris Cité, Paris 75013, France \\ ${ }^{4}$ Université Côte d'Azur, IRD, CNRS, Observatoire de la Côte d'Azur, Géoazur, Sophia Antipolis 06304, France \\ ${ }^{5}$ California Institute of Technology, Seismological Laboratory, Pasadena, CA 91125, USA \\ ${ }^{6}$ Gran Sasso Science Institute, L'Aquila 67100, Italy \\ ${ }^{7}$ INFN, Laboratori Nazionali del Gran Sasso, Assergi, L'Aquila 67100, Italy \\ ${ }^{8}$ Department of Physics, University of Florida, Gainesville, FL 32611, USA
}

Accepted 2018 October 17. Received 2018 October 12; in original form 2017 October 18

\begin{abstract}
SUMMAR Y
As soon as an earthquake starts, the rupture and the propagation of seismic waves redistribute masses within the Earth. This mass redistribution generates in turn a long-range perturbation of the Earth gravitational field, which can be recorded before the arrival of the direct seismic waves. The recent first observations of such early signals motivate the use of the normal mode theory to model the elastogravity perturbations recorded by a ground-coupled seismometer or gravimeter. Complete modelling by normal mode summation is challenging due to the very large difference in amplitude between the prompt elastogravity signals and the direct $P$-wave signal. We overcome this problem by introducing a two-step simulation approach. The normal mode approach enables a fast computation of elastogravity signals in layered self-gravitating Earth models. The fast and accurate computation of gravity perturbations indicates instrument locations where signal detection may be achieved, and may prove useful in the implementation of a gravity-based earthquake early warning system.
\end{abstract}

Key words: Time variable gravity; Transient deformation; Numerical solutions; Theoretical seismology.

\section{INTRODUCTION}

During and after large earthquakes, changes in the gravitational field of the Earth arise from a redistribution of internal masses, at the Earth surface and in depth. Static changes have been reported from before to long after great earthquakes such as the 2003 M8.0 Tokachi-oki earthquake, using data recovered by groundbased superconducting gravimeters (Imanishi 2004). The satellite mission GRACE (Tapley et al. 2004) also recorded gravity changes following the 2004 M9.0-9.3 Sumatra-Andaman earthquake (Han et al. 2006), the 2010 M8.8 Maule earthquake (Han et al. 2010; Heki \& Matsuo 2010) and the 2011 M9.1 Tohoku-oki earthquake (Matsuo \& Heki 2011). In terms of modelling, Okubo (1991, 1992) developed analytical expressions of the static gravity changes due to a point dislocation and faulting of a rectangular finite plane, buried in a homogeneous half-space medium. Sun et al. (2009) later developed the coseismic Green's functions of gravity changes in a spherically symmetric Earth model, at the deformed Earth surface and in space. De Linage et al. (2009) modelled the static perturbation of the gravitational potential induced by the Sumatra-Andaman earthquake, using normal mode summation.

In addition to static gravity effects, time-dependent density perturbations due to seismic waves induce a dynamic long-range perturbation of the gravitational field, during the seismic rupture. Pieces of evidence of such transient perturbations, observed before the arrival of the direct seismic waves following the M9.1 Tohoku-oki earthquake, have been recently reported in Montagner et al. (2016) and Vallée et al. (2017). Harms et al. (2015) first derived an analytical solution of dynamic gravity changes generated by a point dislocation in a homogeneous full-space model. Harms (2016) later provided a dynamic solution of gravity perturbations produced by a dislocation buried in a homogeneous half-space. While that study does not account for the Earth's sphericity and neglects self-gravity effects, it can predict perturbations to distances up to hundreds of kilometres from the epicentre. However, neglecting the coupling 
between gravity and elasticity prevents applying those solutions to simulate data recorded by ground-coupled inertial sensors.

Gravity perturbations ahead of the seismic waves indeed act in turn as body forces that induce additional deformation of the elastic Earth (Heaton 2017; Vallée et al. 2017). A ground-based seismometer therefore records a gravity-induced inertial motion in addition to the gravity perturbation before the first seismic wave arrival: we refer to the overall signal as prompt elastogravity perturbation. Vallée et al. (2017) computed the elastogravity signals induced by the Tohoku earthquake in a layered, non-self-gravitating Earth model, using a three-stage procedure based on the AXITRA code (Cotton \& Coutant 1997). Numerical solutions for the propagation of elastic waves in layered self-gravitating Earth models have been developed since the introduction of normal mode theory in seismology (Alterman et al. 1959; Takeuchi \& Saito 1972; Chaljub \& Valette 2004; Al-Attar \& Woodhouse 2008) and offer a potential direct access to the elastogravity signals. However, none of the previous modelling work based on normal mode theory including self-gravitation has given attention to the part of the seismograms that precedes the first direct $P$-wave arrival. The purpose of this paper is to fill that gap. We show that the task is not trivial, due to the very large difference in amplitude between the prompt elastogravity signals and the direct $P$-wave signal. To overcome the challenge, we introduce a two-step simulation approach.

Normal mode simulations validate the aforementioned analytical formulations when self-gravitation is ignored, reproduce the numerical results from Vallée et al. (2017) while assessing the role of self-gravitation and enable a faster computation of both the gravity perturbation and the induced motion.

\section{ELASTOGRAVITY RESPONSE OFA SEISMOMETER}

\subsection{Fundamental equations}

Let $\rho_{0}$ and $\phi_{0}$ denote the initial density distribution and gravitational potential of a spherically symmetric Earth model. Let also denote the corresponding gravitational acceleration

$\mathbf{g}_{0}=-\nabla \phi_{0}=-\frac{\partial \phi_{0}}{\partial r} \boldsymbol{e}_{r}=-\mathrm{g}_{0} \boldsymbol{e}_{\boldsymbol{r}}$

where $\boldsymbol{e}_{\boldsymbol{r}}$ is the upward unit vector. The gravitational potential obeys Poisson's equation

$\nabla^{2} \phi_{0}=\frac{\partial^{2} \phi_{0}}{\partial r^{2}}+\frac{2}{r} \frac{\partial \phi_{0}}{\partial r}=4 \pi G \rho_{0}$,

where $G$ is Newton's gravitational constant.

Let us consider the displacement field $\mathbf{s}$ due to a point source $\boldsymbol{f}$. Hereafter, the ${ }_{1}$ subscript refers to the perturbed state of a parameter, and the ${ }^{\mathrm{L}}$ and ${ }^{\mathrm{E}}$ superscripts describe Lagrangian and Eulerian representations, respectively. In a self-gravitating Earth, the displacement field generated by seismic wave propagation induces gravity perturbations $\mathbf{g}_{1}$, which in turn disturb the displacement field:

$$
\left\{\begin{aligned}
\rho_{0} \ddot{\mathbf{s}} & =\nabla \cdot \sigma_{1}{ }^{\mathrm{E}}+\rho_{1}{ }^{\mathrm{E}} \mathbf{g}_{\mathbf{0}}+\boldsymbol{f}+\rho_{0} \mathbf{g}_{\mathbf{1}}{ }^{\mathrm{E}}, \\
\nabla^{2} \phi_{1}^{\mathrm{E}} & =\nabla \cdot \mathrm{g}_{1}{ }^{\mathrm{E}}=4 \pi G \rho_{1}^{\mathrm{E}}, \\
\rho_{1}{ }^{\mathrm{E}} & =-\nabla \cdot\left(\rho_{0} \mathbf{s}\right),
\end{aligned}\right.
$$

where $\sigma_{1}{ }^{\mathrm{E}}$ denotes the perturbed stress tensor and $f$ the point source, $\boldsymbol{f}=-\mathbf{M} \cdot \nabla \delta\left(\boldsymbol{r}-\boldsymbol{r}_{\mathbf{s}}\right) H\left(t-t_{\mathrm{s}}\right)$ with $\mathbf{M}$ the moment tensor of the source and $H$ the Heaviside step function (Dahlen \& Tromp 1998).

In eq. (3) above, we assume a hydrostatic initial stress. We do not consider the effect of the Earth's rotation, due to its negligible influence on prompt elastogravity signals in the studied frequency range (2.0-30.0 mHz). According to section 4.4 of Dahlen \& Tromp (1998), the Coriolis force can indeed be neglected for periods much shorter than $1 \mathrm{~d}$. Simulations with geocentric correction included do not lead to significantly different results, thus Earth's ellipticity is also neglected.

Topography and 3-D heterogeneity effects are also neglected in what follows. Their effects on prompt elastogravity signals should however be limited, since the gravity perturbations are not expected to be greatly affected by local heterogeneities. Indeed, the gravity perturbations originate from the volume average, weighted by the distance to the instrument, of the time-dependent density changes due to seismic waves:

$$
\begin{aligned}
\phi_{1}^{\mathrm{E}}(\boldsymbol{r}, t)=G & \int_{V} \frac{\rho_{0}(\boldsymbol{x}) \nabla \cdot \mathbf{s}(\boldsymbol{x}, t)}{\|\boldsymbol{r}-\boldsymbol{x}\|} \mathrm{d}^{3} \boldsymbol{x} \\
& +G \int_{V} \frac{\mathbf{s}(\boldsymbol{x}, t) \cdot \nabla \rho_{0}(\boldsymbol{x})}{\|\boldsymbol{r}-\boldsymbol{x}\|} \mathrm{d}^{3} \boldsymbol{x},
\end{aligned}
$$

where the first term represents the volumetric density perturbation due to dilatation and compression, and the second term gives the perturbation due to the displacement of pre-existing density gradients. The integration over a large volume thus lowers the significance of locally perturbed elastic fields.

\subsection{Response of a seismometer}

We aim to model the complete elastogravity response of a seismometer to an earthquake, prior to the first direct seismic wave arrival (here a 'direct' wave denotes a wave that originates at the earthquake source, in contrast to waves induced by the gravity perturbations). Following Dahlen \& Tromp (1998), the response of a recording instrument must account for gravitational effects in addition to the inertial acceleration of its housing. Thus, we write the momentum equation of its sensing element $\ddot{\xi}=\mathbf{F}+\mathbf{g}_{\mathbf{1}}{ }^{\mathrm{L}}$, where $\boldsymbol{\xi}$ represents the displacement of the sensing element, $\mathbf{F}$ the electromechanical feedback and $\mathbf{g}_{1}{ }^{\mathrm{L}}$ the perturbed gravitational acceleration. The instrument displacement $\boldsymbol{\xi}$ can be decomposed into two terms, $\boldsymbol{\xi}=\mathbf{s}+\boldsymbol{v}$, with $\mathbf{s}$ the displacement of the ground below the station and $\boldsymbol{v}$ the displacement of the sensing element with respect to the instrument housing. Adjusting $\mathbf{F}$ such that the sensing element does not move relatively to its housing $(\boldsymbol{v}=\mathbf{0})$, the instrument records

$\mathbf{F}=\ddot{\xi}-\mathbf{g}_{1}{ }^{\mathrm{L}}=\ddot{\mathbf{s}}-\left(\mathbf{g}_{1}^{\mathrm{E}}+\mathbf{s} \cdot \nabla \mathbf{g}_{0}\right)$.

From eqs (1) and (2), the gradient of the gravitational acceleration can be written as

$\nabla \mathrm{g}_{0}=\left(4 \pi G \rho_{0}-2 \mathrm{~g}_{0} / r\right) \boldsymbol{e}_{\boldsymbol{r}}$.

The three different terms in eq. (5) account respectively for (1) the ground motion, including the gravity-induced ground motion before $P$-wave arrival time, (2) the gravity perturbation and (3) the coupling to the static gravity field (apparent surface-mass perturbation and free-air gradient). It will be shown that the coupling terms are negligible compared to the gravity perturbation and the induced motion, and the recording can be approximated as $\mathbf{F} \simeq \ddot{\mathbf{s}}-\mathbf{g}_{1} \mathrm{E}$. 

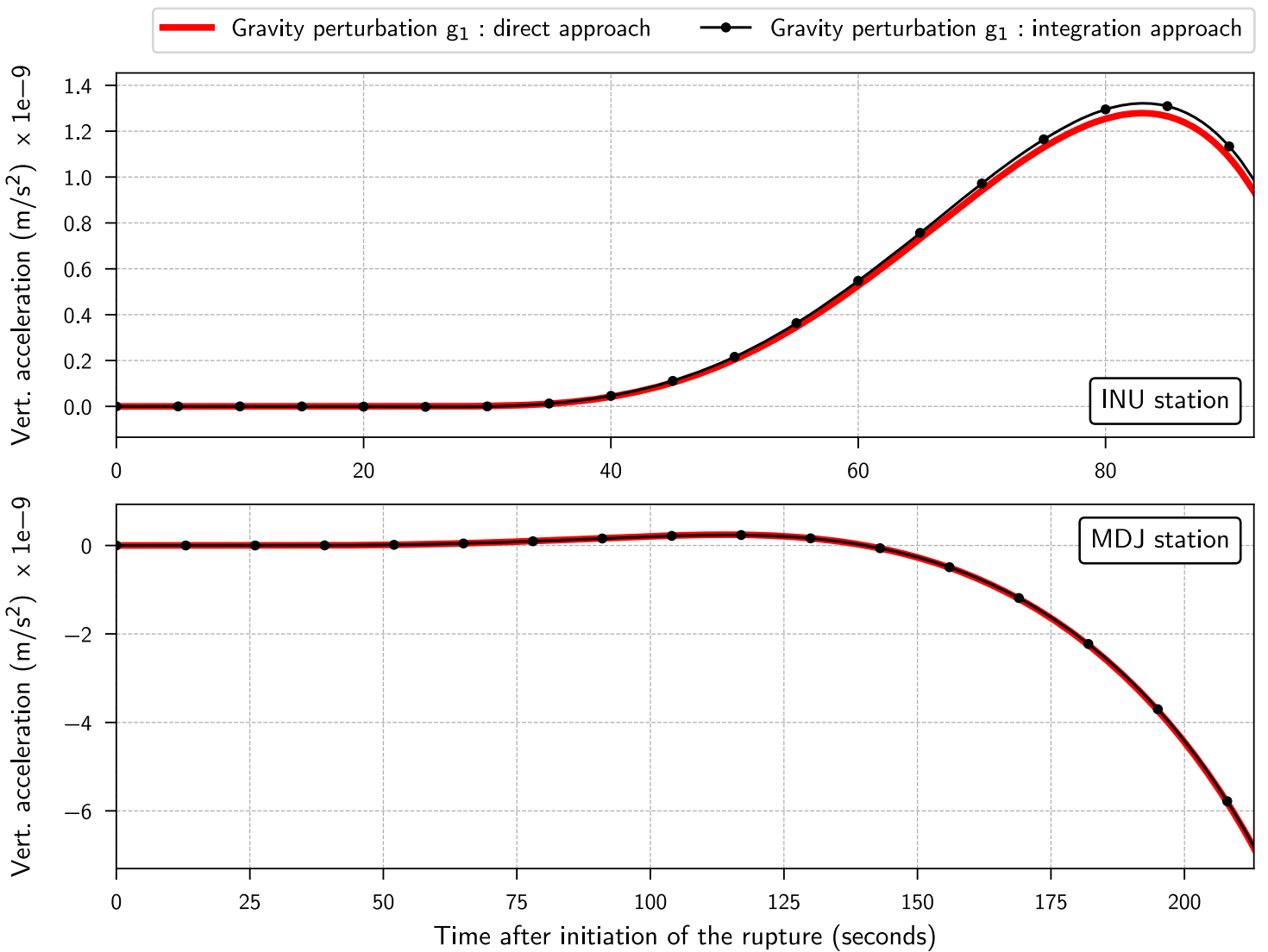

Figure 1. Vertical gravity perturbations $g_{1}$ induced by the M9.1 Tohoku earthquake at INU (top panel) and MDJ (bottom panel) locations, computed in a homogeneous solid sphere. Red lines represent perturbations computed through a straightforward normal mode summation. Black dotted lines represent perturbations computed with an indirect, integration approach. Seismograms are truncated at $P$-wave arrival time.

\section{NORMAL MODE SIMULATIONS OF ELASTOGRAVITY SIGNALS}

\subsection{Spherical-harmonic decomposition}

To compute the prompt elastogravity response of the Earth to an earthquake, we use a spherical-harmonic representation of the displacement vector $\mathbf{s}$ and the gravitational potential $\phi$ (Pekeris \& Jarosch 1958; Alterman et al. 1959):

$$
\left\{\begin{aligned}
\mathbf{s}_{\ell m}= & {\left[\mathrm{U}(r) \boldsymbol{e}_{\boldsymbol{r}}+\mathrm{V}(r) k^{-1} \nabla_{\mathbf{1}}\right.} \\
& \left.-\mathrm{W}(r) k^{-1}\left(\boldsymbol{e}_{\boldsymbol{r}} \times \nabla_{\mathbf{1}}\right)\right] \mathcal{Y}_{\ell m}(\theta, \varphi), \\
\phi_{\ell m}= & \mathrm{P}(r) \mathcal{Y}_{\ell m}(\theta, \varphi),
\end{aligned}\right.
$$

where $\mathcal{Y}_{\ell m}$ is the spherical harmonic of a given $(n, \ell, m)$ mode and $k=\sqrt{\ell(\ell+1)} \cdot \nabla_{1}$ and $\left(\boldsymbol{e}_{r} \times \nabla_{1}\right)$ are the surface gradient and curl on the sphere:

$$
\left\{\begin{aligned}
\nabla_{1} & =\boldsymbol{e}_{\theta} \partial_{\theta}+\boldsymbol{e}_{\boldsymbol{\phi}}(\sin \theta)^{-1} \partial_{\varphi}, \\
\left(\boldsymbol{e}_{\boldsymbol{r}} \times \nabla_{\mathbf{1}}\right) & =-\boldsymbol{e}_{\theta}(\sin \theta)^{-1} \partial_{\varphi}+\boldsymbol{e}_{\boldsymbol{\phi}} \partial_{\theta} .
\end{aligned}\right.
$$

$\boldsymbol{e}_{\theta}$ and $\boldsymbol{e}_{\phi}$ are the unit vectors pointing into south and east directions, respectively. $\mathrm{U}(r), \mathrm{V}(r), \mathrm{W}(r)$ and $\mathrm{P}(r)$ are radial eigenfunctions and only depend on the radial position, and describe respectively the radial and the two transverse displacements, and the gravitational potential.

\subsection{Numerical parameters}

In the following sections, we adopt a self-gravitating isotropic PREM model with no ocean, and compute its eigenfrequencies and eigenfunctions using the Mineos package (Woodhouse 1988). Overtones are summed up to $\ell=2695$ and $200 \mathrm{mHz}$, and progressively tapered from 100 to $200 \mathrm{mHz}$.

In order to validate the analytical solutions, we also compute the free oscillations of a non-self-gravitating, homogeneous solid sphere of density $2700 \mathrm{~kg} / \mathrm{m}^{3}, P$-wave velocity $\alpha=6.4 \mathrm{~km} / \mathrm{s}$ and $S$-wave velocity $\beta=3.7 \mathrm{~km} / \mathrm{s}$ (Takeuchi \& Saito 1972). Overtones are summed up to $\ell=1174$ and $100 \mathrm{mHz}$, and progressively tapered from 50 to $100 \mathrm{mHz}$.

We present results focused on elastogravity perturbations induced by the $M 9.1$ Tohoku-oki earthquake $\left(37.52^{\circ} \mathrm{N}, 143.05^{\circ} \mathrm{E}\right)$, modelled at GEOSCOPE INU station $\left(35.35^{\circ} \mathrm{N}, 137.029^{\circ} \mathrm{E}\right)$, IC MDJ station $\left(44.62^{\circ} \mathrm{N}, 129.59^{\circ} \mathrm{E}\right)$ and GGP KA station $\left(36.43^{\circ} \mathrm{N}\right.$, $\left.137.308^{\circ} \mathrm{E}\right)$ locations. Source rupture is described with angles (strike, dip, rake $)=\left(203^{\circ}, 10^{\circ}, 88^{\circ}\right)$ and we adopt the triangular moment-rate function from the Global Centroid Moment Tensor project (Ekström et al. 2012) as source time function. Elastogravity perturbations are bandpass filtered between $2.0 \mathrm{mHz}$ (Butterworth, 2 poles, causal) and $30.0 \mathrm{mHz}$ (Butterworth, 6 poles, causal) in order to agree with instrumental and seismic noise limitations. 

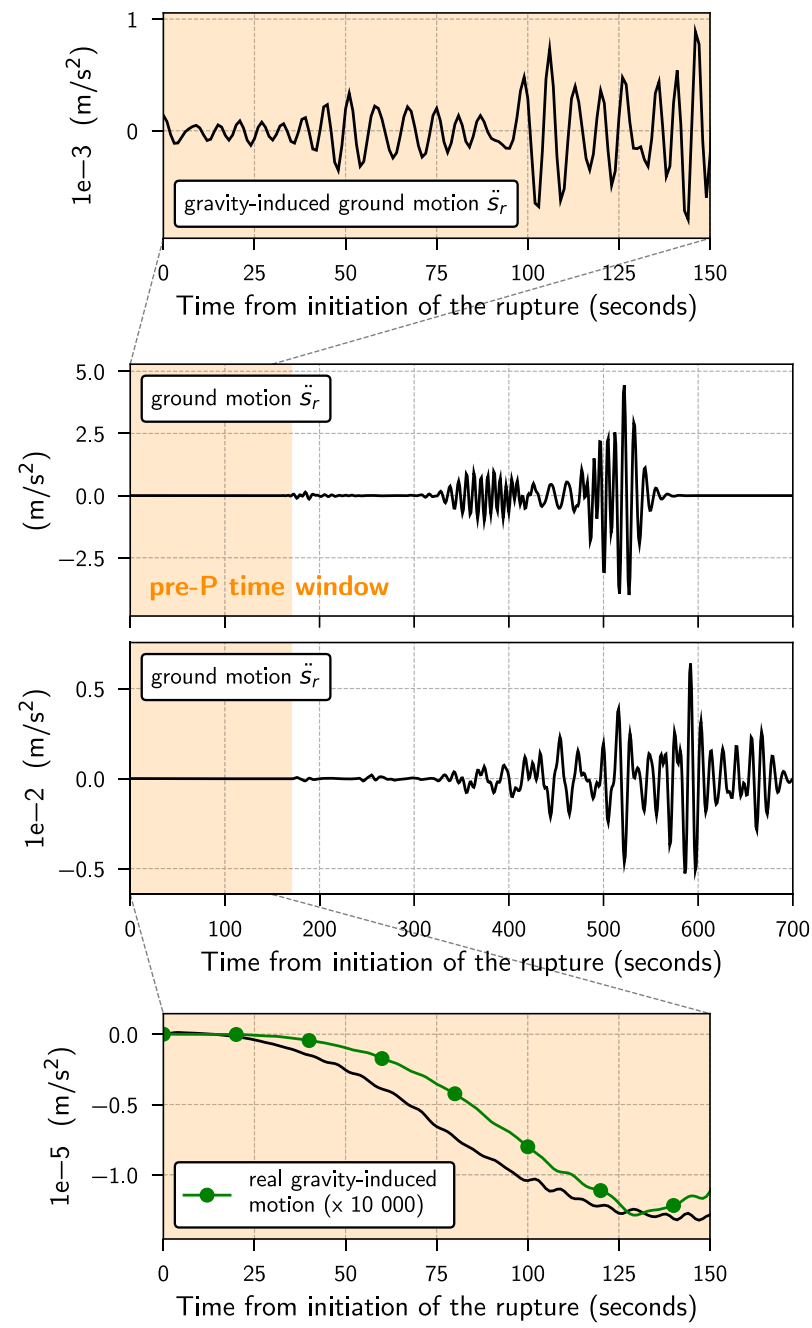
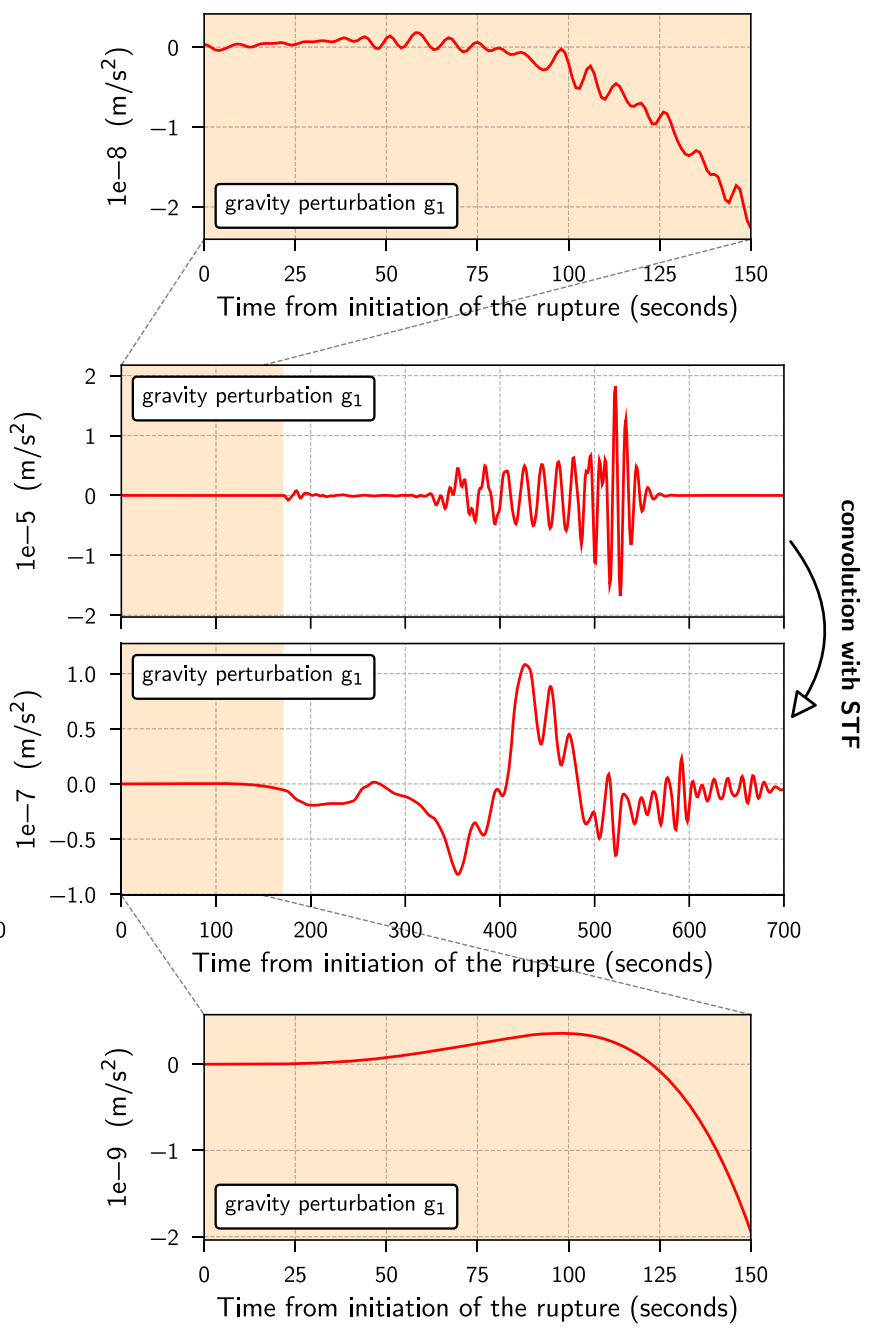

Figure 2. Non-convergence of the direct computation of the gravity-induced motion. The ground motion seismograms are plotted in the left-hand panel, and the gravity perturbations $g_{1}$ in the right-hand panel. (Top rows) Seismograms obtained through a straightforward mode summation at station MDJ, in response to a step-function source. Numerical oscillations prevail in the entire pre- $P$ time window for the gravity-induced ground motion (top left inset). (Bottom rows) Seismograms after convolution with a triangular moment-rate source time function. Due to the very large difference in amplitude between the gravity-induced motion $\left(\sim 10^{-9} \mathrm{~m} / \mathrm{s}^{2}\right)$ and the following direct seismic waves $\left(\sim 10^{-3} \mathrm{~m} / \mathrm{s}^{2}\right)$, the straightforward gravity-induced ground motion (black curve) does not converge towards the real induced motion (green curve), computed with a two-step approach (bottom left inset). On the contrary, the gravity perturbation never exceeds $10^{-7} \mathrm{~m} / \mathrm{s}^{2}$, such that the prompt gravity perturbation converges in the pre- $P$ time window.

\subsection{Computation of the gravity perturbation}

Vallée et al. (2017) computed the gravity perturbation $\mathbf{g}_{1}$ in a nonself-gravitating Earth model using the AXITRA code. They followed an indirect integration approach: first, the direct displacement field induced by the seismic wave propagation is computed on a grid centred around the epicentre location. Then, the gravity perturbation $\mathbf{g}_{1}$ is computed from the integration of the displacement field through the gradient of eq. (4).

We compute the gravity perturbation $\mathbf{g}_{1}$ with a direct, normal mode based approach through the following summation:

$\mathbf{g}_{1}^{\mathrm{E}}(\boldsymbol{r}, t)=-\sum_{i} \mathbf{M}: \boldsymbol{\epsilon}_{\boldsymbol{i}}\left(\boldsymbol{r}_{\mathbf{s}}\right) \dot{m}(t) * \frac{1-\cos \left(\omega_{i} t\right)}{\omega_{i}{ }^{2}} \boldsymbol{\Gamma}_{\boldsymbol{i}}(\boldsymbol{r})$,

where $\boldsymbol{r}=(r, \theta, \varphi)$ are the instrument spherical coordinates, and $\omega_{i}$ is the eigenfrequency of a given mode $i=(n, \ell, m)$ (Gilbert 1971). The double dot product between the moment and strain tensors forms the excitation coefficient $\mathbf{M}: \boldsymbol{\epsilon}$, estimated at source location $\boldsymbol{r}_{\mathbf{s}}$. The asterisk $*$ indicates convolution by the moment-rate source time function $\dot{m}(t)$. The acceleration operator $\boldsymbol{\Gamma}$ comprises the three components of the gravity perturbation:

$\boldsymbol{\Gamma}_{\ell m}(r, \theta, \varphi)=\left[\dot{\mathrm{P}}(r) \boldsymbol{e}_{\boldsymbol{r}}+\mathrm{P}(r) r^{-1} \nabla_{\mathbf{1}}\right] \mathcal{Y}_{\ell m}(\theta, \varphi)$.

The consistency between the integration approach and the direct approach is presented in Fig. 1, in the non-self-gravitating, homogeneous solid sphere. Since the integration approach imposes the computation of thousands of intermediate seismograms, the direct approach is significantly faster.

\subsection{Computation of the gravity-induced ground motion}

\subsubsection{Two-step approach}

The gravity-induced ground motion $\ddot{\mathbf{s}}$ should be computed with a direct, normal mode based approach through the summation:

$\ddot{\mathbf{s}}(r, \theta, \varphi, t)=\sum_{i} \mathbf{M}: \boldsymbol{\epsilon}_{\boldsymbol{i}}\left(\boldsymbol{r}_{\mathbf{s}}\right) \dot{m}(t) * \cos \left(\omega_{i} t\right) \mathcal{A}_{\boldsymbol{i}}(r, \theta, \varphi)$ 


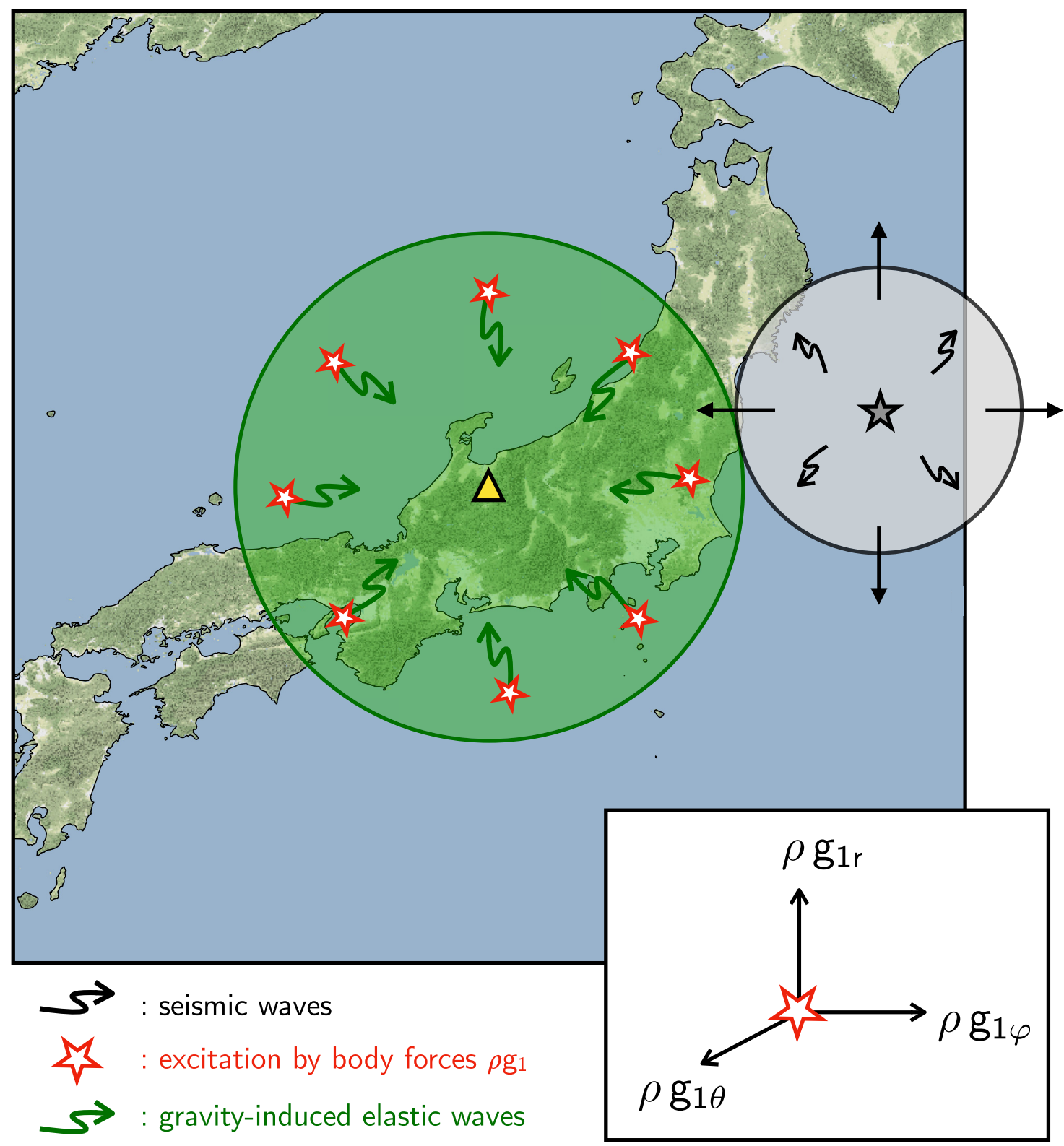

Figure 3. Two-step computation of the gravity-induced ground motion. The earthquake rupture (black star) gives rise to seismic wave propagation (black circle). The mass redistribution in turn generates gravity perturbations $\mathbf{g}_{\mathbf{1}}$ (red stars) in the whole medium ahead of the seismic wave front. In the green volume the gravity perturbations act as body forces, and generate elastic waves that will arrive at the station (yellow triangle) before the first direct seismic wave. These secondary elastic waves are summed into the gravity-induced motion at the station.

and corresponding operator $\mathcal{A}$ :

$$
\begin{aligned}
\mathcal{A}_{\ell m}(r, \theta, \varphi)=[ & \mathrm{U}(r) \boldsymbol{e}_{r}+\mathrm{V}(r) k^{-1} \nabla_{\mathbf{1}} \\
& \left.-\mathrm{W}(r) k^{-1}\left(\boldsymbol{e}_{\boldsymbol{r}} \times \nabla_{\mathbf{1}}\right)\right] \mathcal{Y}_{\ell m}(\theta, \varphi) .
\end{aligned}
$$

However, the immense amplitude difference between the gravityinduced inertial acceleration $\left(\sim 10^{-9} \mathrm{~m} / \mathrm{s}^{2}\right.$ at $\left.1000 \mathrm{~km}\right)$ and the following impulsive direct seismic waves $\left(\sim 10^{-3} \mathrm{~m} / \mathrm{s}^{2}\right.$ at $\left.1000 \mathrm{~km}\right)$ leads to numerical oscillations that mask our signal of interest before the direct $P$ arrival (Fig. 2). Such oscillations are very small and not a nuisance in gravity perturbation seismograms $\mathbf{g}_{\mathbf{1}}$, due to a smaller difference in amplitude between the pre- $P$ time window $\left(\sim 10^{-9} \mathrm{~m} / \mathrm{s}^{2}\right)$ and the post- $P$ time window $\left(\sim 10^{-7} \mathrm{~m} / \mathrm{s}^{2}\right)$. The gravity-induced motion $\ddot{s}$ therefore cannot be computed with a straightforward mode summation. We instead follow a procedure similar to the three-stage method proposed in Vallée et al. (2017), which consists here in only two steps (see Fig. 3 for a schematic).

To obtain the gravity-induced ground motion, we compute the elastic response of the medium surrounding the station to the gravity perturbations induced by the earthquake. Since we aim to reconstruct the inertial motion prior to the direct seismic wave arrival, we only need to consider the portion of the medium that contributes to the gravity-induced signal before the $P$-wave arrival time, that is, the volume shown in green in Fig. 3, shrinking with time as the $P$-wave propagates. We thus define a hemispheric grid centred at the instrument location, with a radius equal to the distance between the instrument and the earthquake source, and grid points every $40 \mathrm{~km}$. An adequate grid sampling that ensures the convergence of the two-step computation is assessed in the next subsection. 

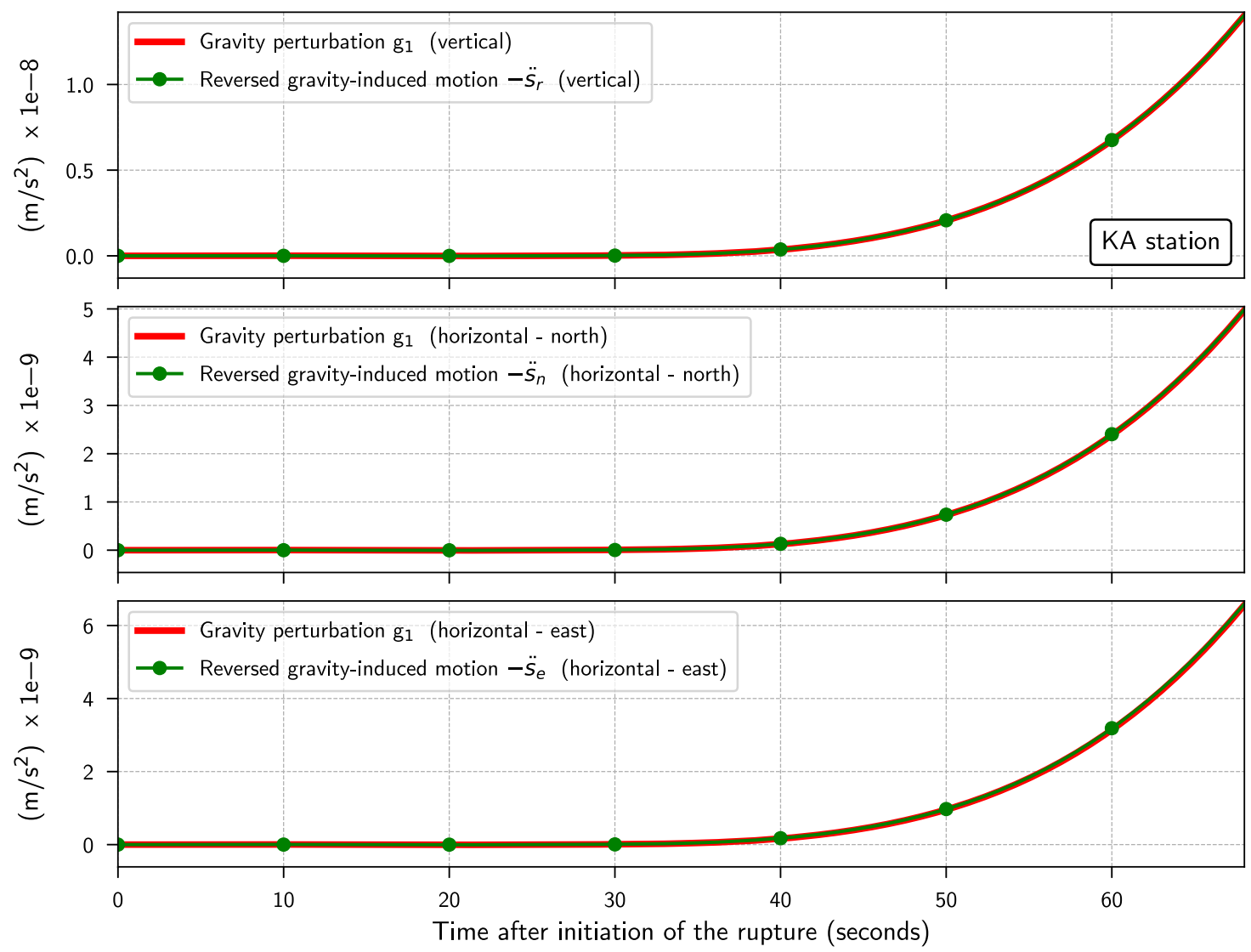

Figure 4. Three-component gravity perturbation $\mathbf{g}_{1}$ (red lines) and reversed gravity-induced motion $-\ddot{\mathbf{s}}$ (green dotted lines) induced by the Tohoku earthquake, recorded at station KA in a homogenous full space. Seismograms are truncated at $P$-wave arrival time. The resulting instrumental record arising from the terms difference is null, in agreement with the theory.

The first step of our computation is thus to compute $\mathbf{g}_{\mathbf{1}}$ in this hemispheric volume:

$\mathbf{g}_{1}\left(\mathbf{r}_{\mathbf{k}}, t\right)=-\sum_{i} \mathbf{M}: \boldsymbol{\epsilon}_{\boldsymbol{i}}\left(\boldsymbol{r}_{\mathbf{s}}\right) \dot{m}(t) * \frac{1-\cos \left(\omega_{i} t\right)}{\omega_{i}{ }^{2}} \boldsymbol{\Gamma}_{\boldsymbol{i}}\left(\mathbf{r}_{\mathbf{k}}\right)$,

where $k$ denotes a grid point and $\mathbf{r}_{\mathbf{k}}$ its corresponding location ahead of the $P$-wave front. Each of these grid points is excited remotely by the gravity perturbation $\mathbf{g}_{1}$ arising from the rupture, acting as a body force $\rho \mathbf{g}_{1}$, and will in turn produce elastic waves that propagate inside the medium and arrive at the instrument location before the direct seismic $P$ wave. The second step of our procedure consists in computing these gravity-induced elastic waves and summing them at the instrument location. The result is the gravity-induced ground motion $\ddot{\mathbf{s}}$ recorded by the instrument:

$\begin{aligned} \ddot{\mathbf{s}}(r, \theta, \varphi, t)=\sum_{i} & \left(\sum_{k} \mathcal{A}_{i}\left(\mathbf{r}_{\mathbf{k}}\right) \cdot \rho_{k} \mathbf{g}_{\mathbf{1}}\left(\mathbf{r}_{\mathbf{k}}, t\right) d V_{k}\right) \\ & * \cos \left(\omega_{i} t\right) \mathcal{A}_{\boldsymbol{i}}(r, \theta, \varphi),\end{aligned}$

where $d V_{k}$ is the volume assigned to each grid point $k$. We point out that the computation of all body force excitations $\rho \mathbf{g}_{1}$ is performed using a single summation based on eq. (13), while Vallée et al. (2017) employed an integration approach based on eq. (4) for each grid point.

We also note that in our PREM model both the body force excitations $\rho \mathbf{g}_{\mathbf{1}}$ and their induced elastic waves are computed in a self-gravitating Earth. However, the two-step induced motion is not a direct solution of the coupling between the equation of motion and Poisson's equation, therefore self-gravitation is not fully accounted for in the computation of the gravity-induced motion. Nonetheless, according to section 4.3.5 of Dahlen \& Tromp (1998) self-gravitation is expected to exert a negligible influence for $\omega \gg$ $\left(4 \pi G \rho_{0}\right)^{1 / 2}$ (i.e. above $0.3 \mathrm{mHz}$ for crustal and upper mantle material), that is, for all frequencies inside the considered frequency range $(2.0-30.0 \mathrm{mHz})$. The influence of self-gravitation on eigenfrequencies, eigenfunctions and seismograms is further discussed in Appendix A (available online).

\subsubsection{Validation of the computation in full-space geometry}

In order to validate the two-step computation of the induced motion and choose the appropriate grid spacing, we compute both the gravity perturbation $\mathbf{g}_{1}$ and the induced ground motion $\ddot{\mathbf{s}}$ in a homogeneous full space. In this model geometry, the induced motion fully cancels the gravity perturbation, as demonstrated in the Supplementary Materials of Vallée et al. (2017). It is thus particularly well suited to assess the convergence of the numerical simulations.

To mimic a homogeneous full space with a normal mode based method, we bury both source and receiver in a homogeneous solid sphere at $1000 \mathrm{~km}$ depth, such that no direct waves reach the free surface before direct $P$-wave arrival time at the station. In a homogeneous full space, the grid of secondary sources becomes purely spherical. Full cancellation of elastogravity terms is verified at station KA during the rupture (Fig. 4). We find that the cancellation is not accurate enough if the grid spacing is larger than $40 \mathrm{~km}$. 

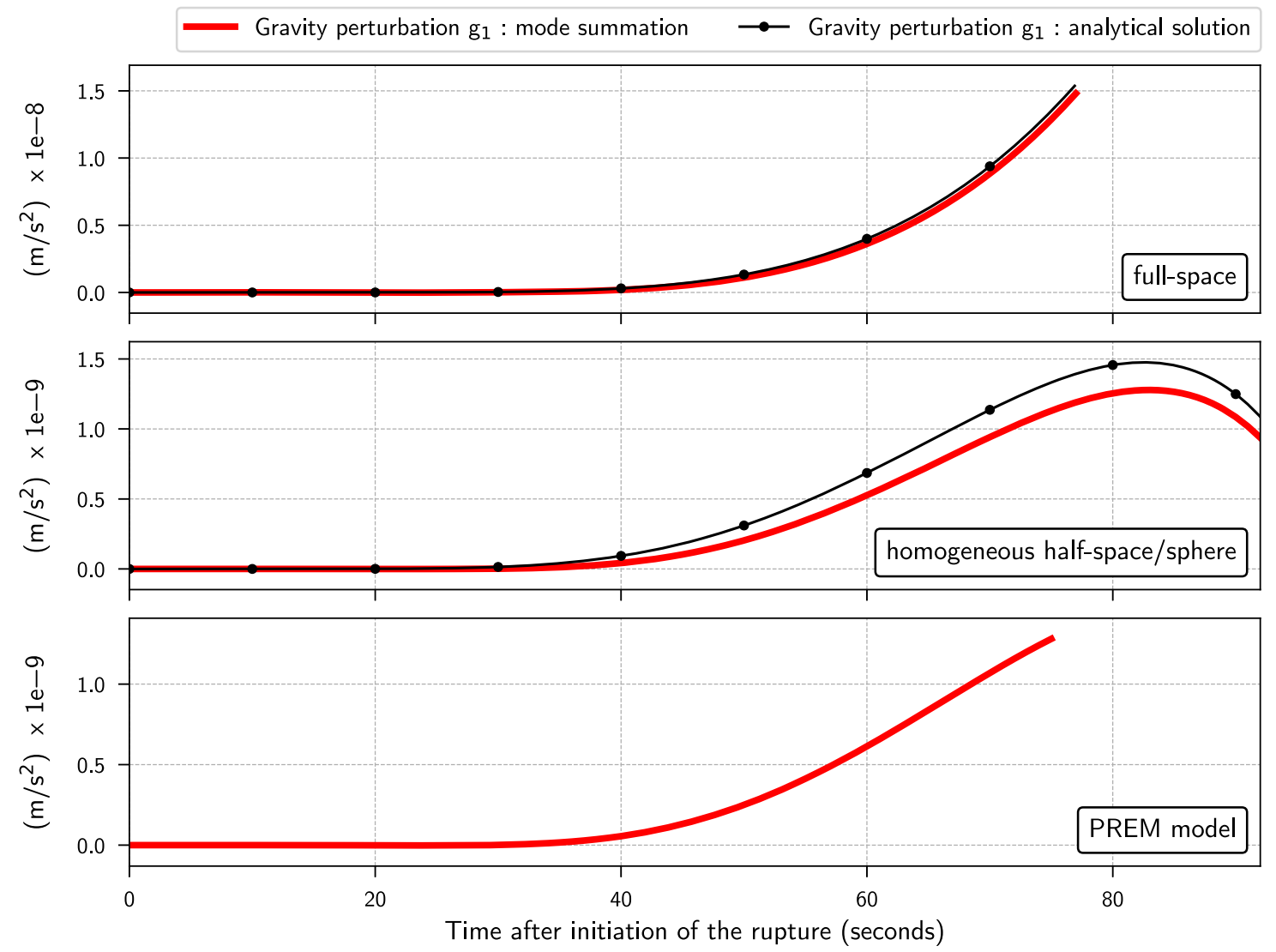

Figure 5. Vertical (upward) gravity perturbations induced by the Tohoku earthquake, recorded at station INU in a full space (top panel), homogeneous solid sphere (middle panel) and self-gravitating PREM model (bottom panel). Red lines represent normal mode seismograms. The black dotted lines are computed from Harms et al. (2015) and Harms (2016) analytical formulations. Seismograms are truncated at $P$-wave arrival time, which depends on model properties.

\section{DISCUSSION}

\subsection{Validation of the analytical formulations}

Harms et al. (2015) derived the following analytical solution of the early gravity potential perturbation in a homogeneous full space, without self-gravitational coupling, induced by a double-couple point source:

$\phi_{1}(r, \theta, \varphi, t)=-R^{\mathrm{P}}(\theta, \varphi) \frac{3 G}{r^{3}} \int_{0}^{t} \int_{0}^{t^{\prime}} m\left(t^{\prime \prime}\right) \mathrm{d} t^{\prime \prime} \mathrm{d} t^{\prime}$,

where $(r, \theta, \varphi)$ are spherical coordinates relative to the hypocentre, $R^{\mathrm{P}}(\theta, \varphi)$ the quadrupolar $P$-wave radiation pattern and $m(t)$ the seismic moment function. The analytical solution is in good agreement with the normal mode gravity perturbation computed in the full-space geometry (Fig. 5, top panel). The homogeneous, half-space analytical solution developed by Harms (2016) shares common patterns with normal mode seismograms computed in a homogeneous sphere (Fig. 5, middle panel). The inclusion of seismic waves reflected by the free surface leads to signal shapes and amplitudes significantly different from the full-space geometry. The computation of gravity perturbations in more complex layered media such as the PREM model (Fig. 5, bottom panel) enables the simulation of realistic seismic wave fronts, with an accurate duration for the pre- $P$ time window.

\subsection{Behaviour of gravity perturbation $\mathrm{g}_{1}$}

The evolution of the gravity perturbation recorded at station MDJ can be explained through a cross-section of the divergence of the displacement field along the azimuth to the station (Fig. 6), in the homogeneous solid sphere. At short times the gravity perturbation is initially positive, due to compressed material below the ruptured area. The compressed materials are located deeper than the dilated materials, thus the projection of their gravity perturbations on the instrument vertical axis is more effective. With the incoming dilating $P, p P$ and $s P$ wave fronts, the dilated areas are progressively drawn closer to the observation point, and the effectiveness of the projection of their gravity perturbations on the instrument vertical axis increases: the gravity perturbation becomes increasingly negative.

\subsection{Three-component elastogravity perturbation}

The normal mode simulations enable to investigate the complete elastogravity perturbations on all three components: the perturbations simulated at station INU in the PREM model are displayed in Fig. 7. The terms representing the coupling to the static gravity field are negligible. The induced motion tends to reduce the amplitude of the overall perturbation, as predicted by Heaton (2017). However, in contrast to the full-space model, in which the direct and induced effects cancel each other perfectly up to the $P$-wave arrival time, the two effects are distinct in the PREM model and an elastogravity perturbation arises well before the arrival of the direct seismic 

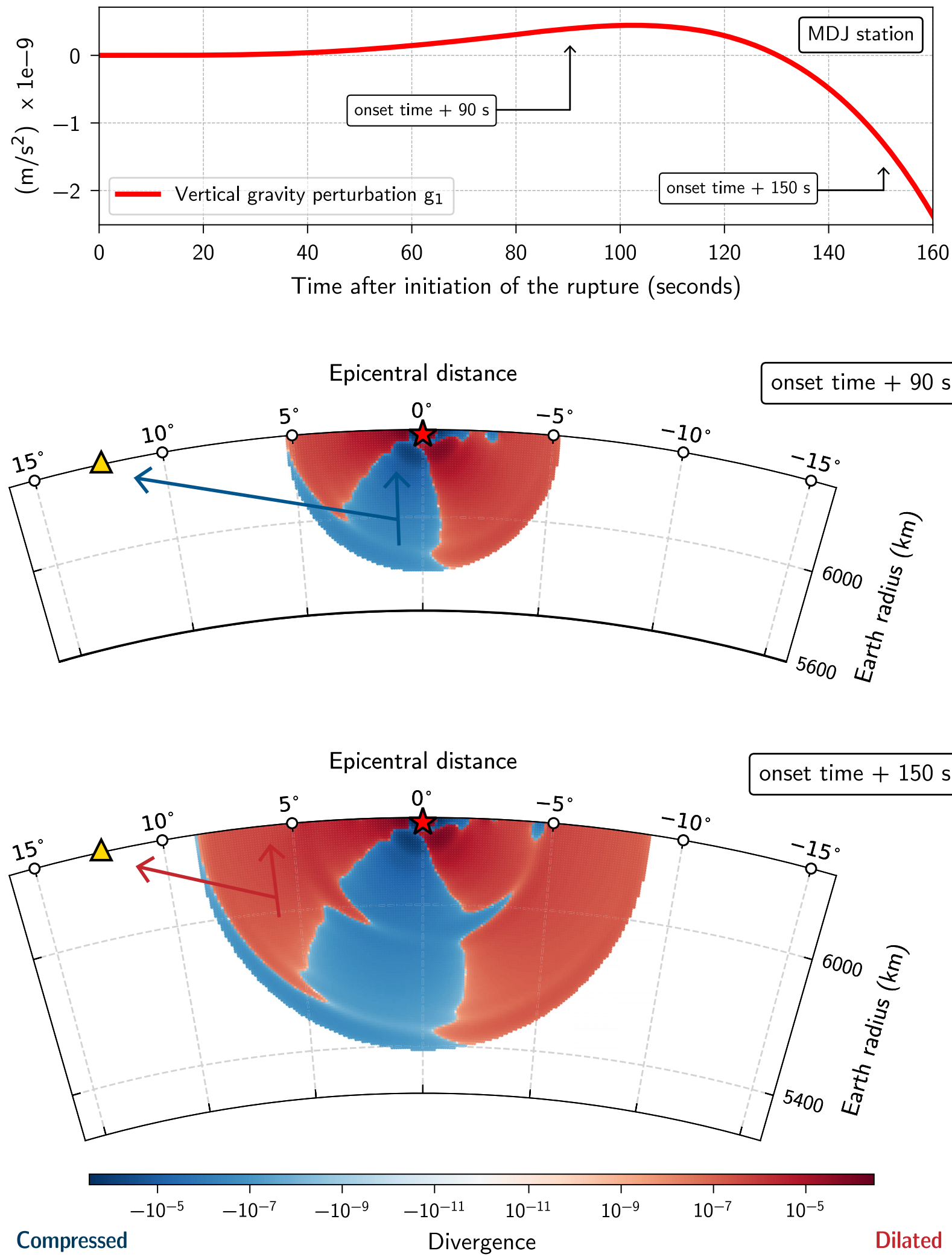

Figure 6. Vertical gravity perturbation $g_{1}$ at station MDJ (top panel) and cross-section of the divergence of the displacement field along azimuth to the station, induced by the Tohoku earthquake $90 \mathrm{~s}$ (middle panel) and $150 \mathrm{~s}$ (bottom panel) after the initiation of the rupture, in a homogeneous solid sphere. The red star represents the earthquake epicentre, the yellow triangle represents MDJ station location. Blue areas are compressed by the seismic wave propagation, red areas are dilated. 

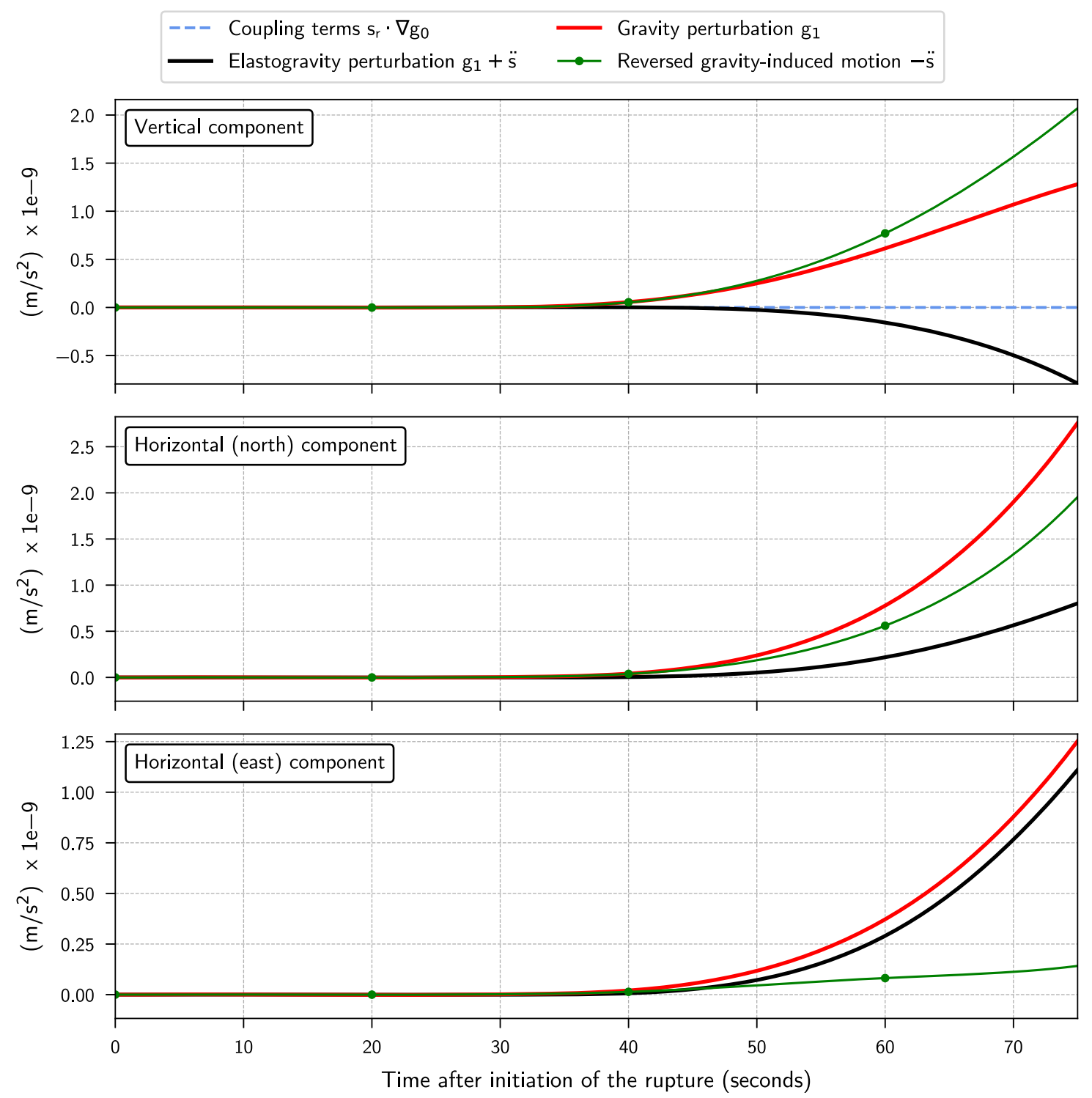

Figure 7. Three-component elastogravity perturbation (black lines), separated into gravity perturbation $\mathbf{g}_{1}$ (red lines) and reversed gravity-induced motion $-\ddot{\mathbf{s}}$ (green lines), induced by the Tohoku earthquake at station INU in the self-gravitating PREM model. Seismograms are truncated at $P$-wave arrival time. Up to $40-50 \mathrm{~s}$ after the initiation of the rupture, the induced motion and the gravity perturbation balance each other out. Coupling terms to the static gravity field (blue dashed line) are negligible.

waves. The amplitude of the perturbations is similar on all three components. However, long-period horizontal recordings are usually noisier than vertical ones, due to their higher sensitivity to tilt effects. An elastogravity detection on horizontal recordings might then prove difficult, but still needs to be investigated. The shape of the elastogravity perturbation differs significantly from the sole gravity perturbation, thus both terms must be taken into account in simulations of elastogravity signals recorded by seismometers or gravimeters.

\subsection{Surface map of gravity perturbation $\mathrm{g}_{1}$}

The fast normal mode computation of gravity perturbations $\mathbf{g}_{1}$ enables to draw perturbation maps such as Fig. 8. The vertical gravity perturbations induced by the Tohoku earthquake at the direct $P$ wave arrival time are displayed in Fig. 8 , in the self-gravitating
PREM model. Corresponding maps for horizontal components can be found in Appendix B (available online).

The surface map confirms several features of the gravity perturbations described in Vallée et al. (2017). First, stations located in the $P$-wave extensional direction ( $\sim$ along azimuth to station MDJ) record strong gravity perturbations ahead of the direct $P$-wave front. These stations record a stronger perturbation when deployed at regional distances from the ruptured area $(1000-2000 \mathrm{~km}$ away from the epicentre), due to the growing density anomaly generated by the rupture itself and the larger volume perturbed by the propagating seismic waves. At further distances (epicentral distances $>$ $2500 \mathrm{~km}$ ) the perturbation starts to decay. Finally, due to the fault plane geometry (thrust mechanism in an east-west subduction context), stations located north of the epicentre display a low gravity perturbation.

Although it only represents a part of the total elastogravity perturbation, surface maps of gravity perturbations $\mathbf{g}_{1}$ can be used as a 


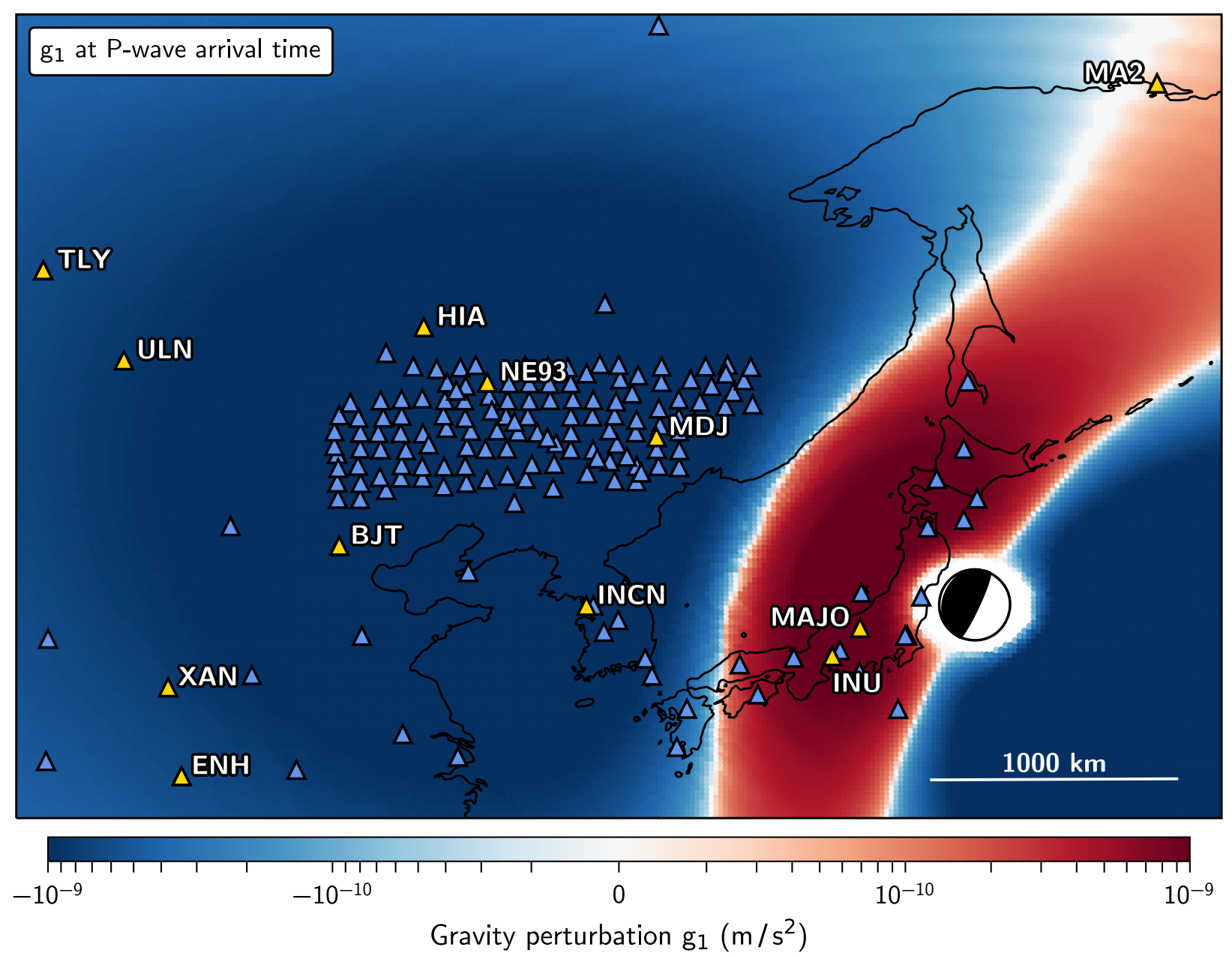

Figure 8. Vertical gravity perturbation $\mathrm{g}_{1}$ induced at the Earth surface by the $M 9.1$ Tohoku-oki earthquake, at $P$-wave arrival time. The focal mechanism locates the earthquake epicentre, and the triangles represent stations from the IRIS database. Yellow triangles are stations with low background seismic noise in the 30 min preceding the event. In the area close to the epicentre the simulations are less reliable due to the very short $P$ traveltimes, and the perturbations are not computed.

proxy for signal observability. We thus fetch data recordings from the IRIS database, and focus on stations with low background seismic noise in the 30 min preceding the event (standard deviation lower than $0.5 \mathrm{~nm} \mathrm{~s}^{-2}$, these stations are indicated by yellow triangles in Fig. 8). The stations that have a strong predicted gravity perturbation (say $>1.0 \mathrm{~nm} / \mathrm{s}^{2}$ ) should be well suited for the detection of elastogravity perturbations.

The set of stations selected by these criteria include most of those used in Vallée et al. (2017) data analysis. The agreement between the observed elastogravity perturbations, AXITRA simulations and normal mode seismograms is shown in Fig. 9. Such agreement confirms that self-gravitation can be neglected in the frequency range of analysis $(2.0-30.0 \mathrm{mHz})$. Moreover, the observed waveforms are accurately modelled with 1-D model-based simulations, meaning that the overlooked topography and 3-D heterogeneities indeed seem to be negligible.

\subsection{Gravity-based earthquake and tsunami early warning}

A fast and robust estimation of the magnitude of large earthquakes is crucial for earthquake and tsunami warning systems, especially for regions close to the rupture area. Tsunami warning centres currently use a magnitude estimate based on seismic waves recorded distantly, unlikely to be available within the first 15 min after the earthquake onset time due to the seismic wave propagation delay. Unfortunately, such time delay is close to the time needed for the first tsunami wave to reach the nearest coastal regions.

Joint seismic and geodetic systems currently under development could provide unsaturated magnitude estimates within a few tens of seconds from the earthquake onset time (Crowell et al. 2016; Ruhl et al. 2017), using local high-rate geodetic data. The detection of prompt elastogravity signals could provide a robust, final estimate of the earthquake magnitude as soon as the rupture stops, based on a sparse network of broad-band seismometers deployed at regional distances (Vallée et al. 2017). Thus, both methods could be used as complementary tools to assess that a large rupture is happening.

However, the sensitivity of present seismometers prevents direct elastogravity signal detection for subduction earthquakes of magnitude much smaller than 9. A change in the detector concept could enable to detect prompt gravity signals induced by smaller magnitude earthquakes, and provide faster warnings and magnitude estimations (Juhel et al. 2018). Valuable seconds could then be saved in comparison to conventional warning systems, which could be exploited to launch automatic prevention systems or enhance personal protective actions (Allen 2013). 


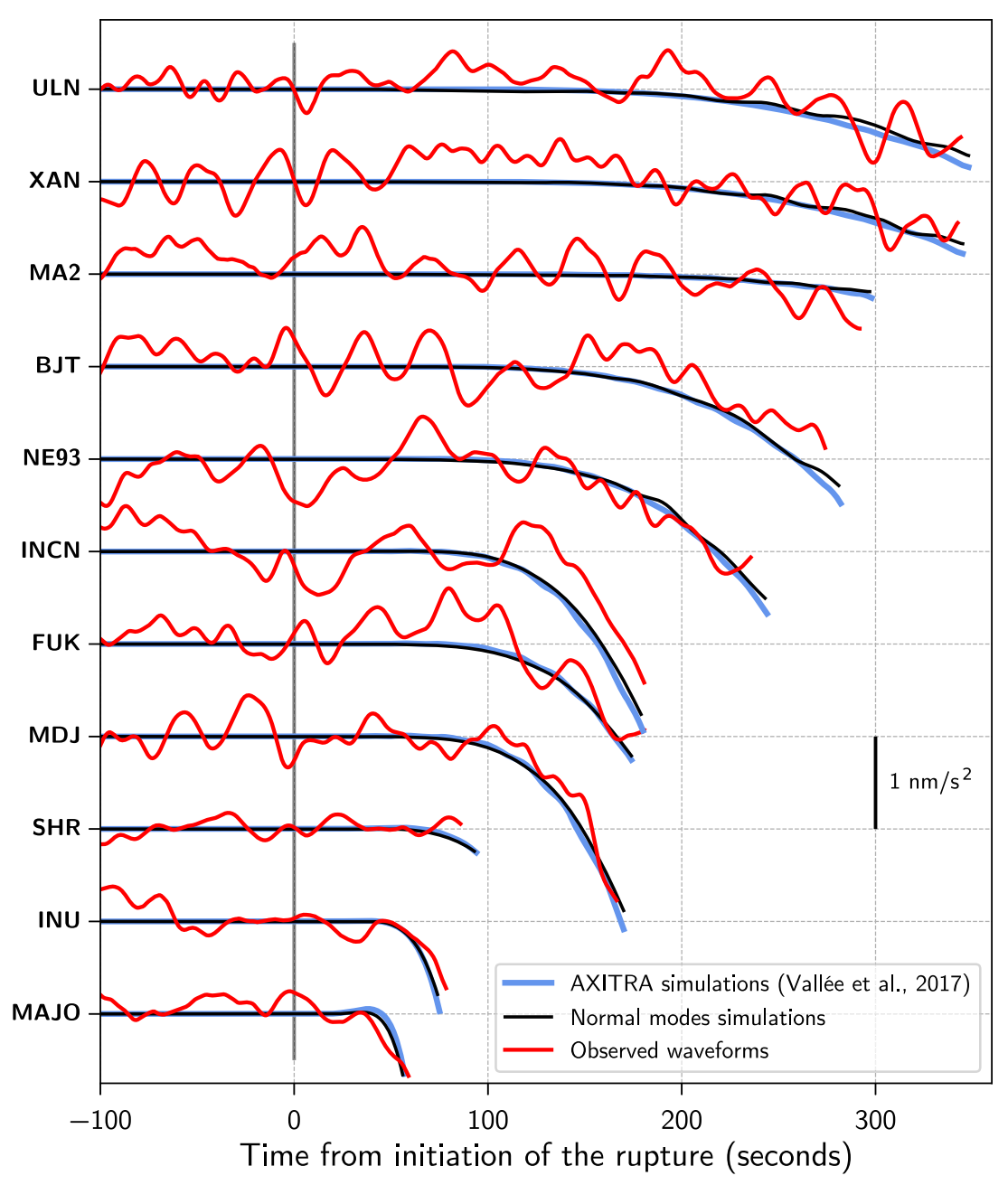

Figure 9. Observed elastogravity perturbations (red lines) recorded by high-quality stations during the Tohoku earthquake, and corresponding numerical simulations. AXITRA simulations (blue lines, Vallée et al. 2017) and normal mode simulations (this study) are in good agreement, and fit the observed waveforms at all stations.

Ultra-sensitive gravity strainmeters are currently being developed for low-frequency gravitational waves detection purposes. Sensors such as torsion-bars antenna (Ando et al. 2010; McManus et al. 2017) or superconducting gravity strainmeters (Moody et al. 2002; Paik et al. 2016) record the gradient of the perturbed gravity field from several sensing masses, attached to a common housing. The background seismic noise is similar in every test mass, and rejected by the differential measurement. Besides this noise reduction, the gravity-induced motion is no longer recorded in such instruments: the cancelling effect observed on seismometers at short times is then avoided. Several instrumental challenges are yet to be overcome, but the sensitivity target of $10^{-15} \mathrm{~Hz}^{-1 / 2}$ at $0.1 \mathrm{~Hz}$ seems within reach (Harms et al. 2015). Such sensitivity is sufficient for the measurement of gravity strain perturbations induced by earthquakes, and would enable the detection of gravity signals at lower magnitudes. An earthquake early warning system based on these gravity strainmeters could then be implemented. Gravity strain is the second integral over time of the gravity gradient, which can be computed by finite difference of gravity perturbation computed at two closely located receivers. The straightforward mode summation from eq. (9) may be beneficial for gravity-based earthquake early warning system, by enabling fast computation of pseudo real-time scenarios of earthquake ruptures.

\section{CONCLUSION}

Ground-based seismometers record the gravity perturbation and gravity-induced ground motion generated by a seismic rupture, before the arrival of the strong direct seismic $P$ wave at the station. In addition to Vallée et al. (2017) simulations, this study provides an alternate way to model elastogravity perturbations. The normal mode based approach enables a direct and faster computation of the gravity perturbation, and a two-step computation of the gravity-induced motion. Its accuracy is confirmed in full-space geometry with the cancellation of elastogravity terms. The importance of the free surface effects on the computation of gravity perturbations, as well as the cancelling effect of the induced motion on the early overall perturbation are confirmed by our simulations. The computation of gravity perturbations validates the analytical solutions, and indicates instrument locations where detection may be achieved. Associated with the current development of high-precision gravity strainmeters, the normal mode computation of gravity perturbations may prove useful in the implementation of a gravity-based earthquake early warning system. 


\section{ACKNOWLEDGEMENTS}

We thank Philippe Lognonné, Yann Capdeville, David Al-Attar and Olivier Coutant for making their codes publicly available, and Thomas Heaton for stimulating discussions. We thank engineers involved in the installation, maintenance and data distribution of broad-band seismic stations. High-quality signals come from the Global Seismograph Network (ASL/USGS 1988), the New China Digital Seismograph Network (ASL/USGS 1992), the National Research Institute for Earth Science and Disaster Resilience/F-net, GEOSCOPE (IPGP/EOST 1982) and the Northeast China Extended Seismic Array (Ni 2009), all publicly available at the IRIS data management center, the Institut de Phys ique du Globe de Paris (IPGP) data center or the F-net data center. We acknowledge the financial support from the UnivEarthS Labex program at Sorbonne Paris Cite (grants ANR-10-LABX-0023 and ANR-11-IDEX-0005-02) and the financial support of the Agence Nationale de la Recherche through the grant ANR-14-CE03-001401. JPM acknowledges the financial support of I.U.F. (Institut universitaire de France). JPA acknowledges funding by the French government through the "Investissements d'Avenir UCAJEDI" project managed by the Agence Nationale de la Recherche (grant ANR-15IDEX-01). Numerical computations were partly performed on the S-CAPAD platform, IPGP, France.

\section{REFERENCES}

Al-Attar, D. \& Woodhouse, J.H., 2008. Calculation of seismic displacement fields in self-gravitating Earth models - applications of minors vectors and symplectic structure, Geophys. J. Int., 175(3), 1176-1208.

Allen, R., 2013. Seismic hazards: seconds count, Nature, 502(7469), 29-31.

Alterman, Z., Jarosch, H. \& Pekeris, C., 1959. Oscillations of the Earth, Proc. R. Soc., A, 80-95.

Ando, M., Ishidoshiro, K., Yamamoto, K., Yagi, K., Kokuyama, W., Tsubono, K. \& Takamori, A., 2010. Torsion-bar antenna for low-frequency gravitational-wave observations, Phys. Rev. Lett., 105(16).

ASL/USGS, 1988. Global Seismographic Network (GSN-IRIS/USGS), International Federation of Digital Seismograph Networks, Other/Seismic Network, doi:10.7914/SN/IU.

ASL/USGS, 1992. New China Digital Seismograph Network, International Federation of Digital Seismograph Networks, Other/Seismic Network, doi: $10.7914 / \mathrm{SN} / \mathrm{IC}$.

Chaljub, E. \& Valette, B., 2004. Spectral element modelling of threedimensional wave propagation in a self-gravitating Earth with an arbitrarily stratified outer core, Geophys. J. Int., 158(1), 131-141.

Cotton, F. \& Coutant, O., 1997. Dynamic stress variations due to shear faults in a plane-layered medium, Geophys. J. Int., 128(3), 676-688.

Crowell, B.W. et al., 2016. Demonstration of the Cascadia G-FAST geodetic earthquake early warning system for the Nisqually, Washington, earthquake, Seismol. Res. Lett., 87(4), 930-943.

Dahlen, F.A. \& Tromp, J., 1998. Theoretical Global Seismology, Princeton University Press.

De Linage, C., Rivera, L., Hinderer, J., Boy, J.-P., Rogister, Y., Lambotte, S. \& Biancale, R., 2009. Separation of coseismic and postseismic gravity changes for the 2004 Sumatra-Andaman earthquake from $4.6 \mathrm{yr}$ of GRACE observations and modelling of the coseismic change by normalmodes summation, Geophys. J. Int., 176(3), 695-714.

Ekström, G., Nettles, M. \& Dziewoński, A., 2012. The global CMT project 2004-2010: centroid-moment tensors for 13,017 earthquakes, Phys. Earth planet. Inter., 200, 1-9.

Gilbert, F., 1971. Excitation of the normal modes of the Earth by earthquake sources, Geophys. J. Int., 22(2), 223-226.

Han, S.-C., Sauber, J. \& Luthcke, S., 2010. Regional gravity decrease after the 2010 Maule (Chile) earthquake indicates large-scale mass redistribution, Geophys. Res. Lett., 37(23).
Han, S.-C., Shum, C., Bevis, M., Ji, C. \& Kuo, C.-Y., 2006. Crustal dilatation observed by GRACE after the 2004 Sumatra-Andaman earthquake, Science, 313(5787), 658-662.

Harms, J., 2016. Transient gravity perturbations from a double-couple in a homogeneous half-space, Geophys. J. Int., 205(2), 1153-1164.

Harms, J., Ampuero, J.-P., Barsuglia, M., Chassande-Mottin, E., Montagner, J.-P., Somala, S.N. \& Whiting, B.F., 2015. Transient gravity perturbations induced by earthquake rupture, Geophys. J. Int., 201(3), $1416-1425$.

Heaton, T.H., 2017. Correspondence: response of a gravimeter to an instantaneous step in gravity, Nat. Commun., 8(1), doi:10.1038/s41467-01701348-z.

Heki, K. \& Matsuo, K., 2010. Coseismic gravity changes of the 2010 earthquake in central Chile from satellite gravimetry, Geophys. Res. Lett., 37(24).

Imanishi, Y., 2004. A network of superconducting gravimeters detects submicrogal coseismic gravity changes, Science, 306(5695), 476-478.

IPGP/EOST, 1982. GEOSCOPE, French Global Network of broad-band seismic stations, doi: 10.18715/GEOSCOPE.C.

Juhel, K. et al., 2018. Earthquake early warning using future generation gravity strainmeters, EarthArXiv, doi:10.31223/osf.io/yqb3a.

Matsuo, K. \& Heki, K., 2011. Coseismic gravity changes of the 2011 Tohoku-oki earthquake from satellite gravimetry, Geophys. Res. Lett., 38(7).

McManus, D., Forsyth, P., Yap, M., Ward, R., Shaddock, D., McClelland, D. \& Slagmolen, B., 2017. Mechanical characterisation of the TorPeDO: a low frequency gravitational force sensor, Class. Quantum Gravity, 34(13), doi:10.1088/1361-6382/aa7103.

Montagner, J.-P., Juhel, K., Barsuglia, M., Ampuero, J.P., Chassande-Mottin, E., Harms, J., Whiting, B.F., Bernard, P., Clévédé, E. \& Lognonné, P., 2016. Prompt gravity signal induced by the 2011 Tohoku-Oki earthquake, Nat. Commun., 7, doi:10.1038/ncomms13349.

Moody, M.V., Paik, H.J. \& Canavan, E.R., 2002. Three-axis superconducting gravity gradiometer for sensitive gravity experiments, Rev. Sci. Instrum., 73(11), 3957-3974.

Ni , J., 2009. Collaborative Research: Northeast China Extended Seismic Array: Deep subduction, mantle dynamics and lithospheric evolution beneath Northeast China, International Federation of Digital Seismograph Networks, Other/Seismic Network, 10, doi:10.7914/SN/YP_2009.

Okubo, S., 1991. Potential and gravity changes raised by point dislocations, Geophys. J. Int., 105(3), 573-586.

Okubo, S., 1992. Gravity and potential changes due to shear and tensile faults in a half-space, J. geophys. Res., 97(B5), 7137-7144.

Paik, H.J., Griggs, C.E., Moody, M.V., Venkateswara, K., Lee, H.M., Nielsen, A.B., Majorana, E. \& Harms, J., 2016. Low-frequency terrestrial tensor gravitational-wave detector, Class. Quantum Gravity, 33(7), doi:10.1088/0264-9381/33/7/075003.

Pekeris, C.L. \& Jarosch, H., 1958. The free oscillations of the Earth, in Contributions in Geophysics in Honor of Beno Gutenberg, pp. 171-192, eds Benioff, H., Ewing, M., .Howell, B.F., Jr \& Press, F., Pergamon Press.

Ruhl, C., Melgar, D., Grapenthin, R. \& Allen, R., 2017. The value of realtime GNSS to earthquake early warning, Geophys. Res. Lett., 44(16), 8311-8319.

Sun, W., Okubo, S., Fu, G. \& Araya, A., 2009. General formulations of global co-seismic deformations caused by an arbitrary dislocation in a spherically symmetric Earth model-applicable to deformed Earth surface and space-fixed point, Geophys. J. Int., 177(3), 817-833.

Takeuchi, H. \& Saito, M., 1972. Seismic surface waves, in Methods in Computational Physics: Advances in Research and Applications, pp. 217295, ed. Bolt, B.A., Elsevier.

Tapley, B.D., Bettadpur, S., Watkins, M. \& Reigber, C., 2004. The gravity recovery and climate experiment: mission overview and early results, Geophys. Res. Lett., 31(9).

Vallée, M., Ampuero, J.P., Juhel, K., Bernard, P., Montagner, J.-P. \& Barsuglia, M., 2017. Observations and modeling of the elastogravity signals preceding direct seismic waves, Science, 358(6367), 1164-1168.

Woodhouse, J.H., 1988. The calculation of the eigenfrequencies and eigenfunctions of the free oscillations of the Earth and the Sun, in Seismological 
Algorithms: Computational Methods and Computer Programs, pp. 321370, eds Doornbos, D., Academic Press.

\section{SUPPORTING INFORMATION}

Supplementary data are available at $G J I$ online.

Figure A1. Dispersion diagram of spheroidal oscillations for an isotropic PREM model (no ocean), with (black dots) and without (orange dots) self-gravitation included. Self-gravitation effects are significant only at low frequencies and angular number $\ell$.

Figure A2. Eigenfunctions $\dot{\mathrm{P}}(r)$ within a homogeneous solid sphere, with (black lines) and without (orange lines) self-gravitation included. These are fundamental modes $(n=0)$ and the horizontal angular wavenumber $\ell$ ranges from 0 to 11 . Self-gravitation effects are significant only at low frequencies. The ${ }_{0} \mathcal{S}_{1}$ spheroidal mode is not computed, since it implies a displacement of the centre of the Earth.

Figure A3. Vertical gravity perturbation induced by the M9.1 Tohoku earthquake, recorded at INU (top panel) and MDJ (bottom panel) stations, in a non-self-gravitating (orange lines) and selfgravitating (black lines) isotropic PREM model (no ocean).
Figure A4. Horizontal (north) gravity perturbation $\mathrm{g}_{1}$ induced at the Earth surface by the M9.1 Tohoku-oki earthquake, at $P$-wave arrival time. The focal mechanism locates the earthquake epicentre, and the triangles represent stations from the IRIS database. Yellow triangles are stations with low background seismic noise in the $30 \mathrm{~min}$ preceding the event. In the area close to the epicentre the simulations are less reliable due to the very short $P$ traveltimes, and the perturbations are not computed.

Figure A5. Horizontal (east) gravity perturbation $g_{1}$ induced at the Earth surface by the $M 9.1$ Tohoku-oki earthquake, at $P$-wave arrival time. The focal mechanism locates the earthquake epicentre, and the triangles represent stations from the IRIS database. Yellow triangles are stations with low background seismic noise in the 30 min preceding the event. In the area close to the epicentre the simulations are less reliable due to the very short $P$ traveltimes, and the perturbations are not computed.

Please note: Oxford University Press is not responsible for the content or functionality of any supporting materials supplied by the authors. Any queries (other than missing material) should be directed to the corresponding author for the article. 